\title{
The CP Stars, an overview: Then and now
}

\author{
Charles R. Cowley ${ }^{1}$ and Donald J. Bord ${ }^{2}$ \\ ${ }^{1}$ Department of Astronomy, University of Michigan, Ann Arbor, MI 48109-1090, USA \\ email: cowley@umich.edu \\ ${ }^{2}$ Department of Natural Sciences, University of Michigan-Dearborn, \\ 4901 Evergreen Rd., Dearborn, MI 48128-1491, USA \\ email: dbord@umd.umich.edu
}

\begin{abstract}
We briefly review the traditional classifications of CP stars. The current availability of large numbers of abundances now make it possible to use multivariate techniques, both to supplement traditional classification methods and probe the abundance patterns. We discuss cluster analysis and correlation matrices for sample material. We review the historical resistance to the notion that CP stars were indeed chemically peculiar. Modern work shows that while these objects do indeed have atmospheric anomalies, they are nevertheless chemically peculiar.

Abundance patterns are an important clue to the origin of the abundance peculiarities. We contrast patterns due to nuclear and chemical differentiation processes. The roAp and related stars show vertical as well as horizontal abundance variations, and abnormal line profiles. Photospheric abundances in these stars are surely abnormal (nonsolar), but as long as the models are uncertain the derived abundances will be very crude.

For more than two decades, observations of CP stars in the X-ray and radio regimes have been made with increasing sensitivity and pointing accuracy. We discuss the current evidence linking magnetic and nonmagnetic $\mathrm{CP}$ stars to sources of galactic $\mathrm{X}$-rays and radio radiation. There seems no doubt that high energy phenomena are associated with, if not produced by, some CP stars. This circumstance admits the possibility that the release of high energy particles (p's, n's and $\alpha$ 's) during such events may initiate nuclear reactions on the surfaces of the CP stars. We briefly reconsider the viability of such processes for producing exotic species like Pm by proton bombardment using recent data for solar and stellar flares.
\end{abstract}

Keywords. Stars: abundances, stars: chemically peculiar, stars: individual: (Vega, Merak, $\alpha$ And, 32 Aqr, $\sigma$ Boo, $\kappa$ Cnc, $\theta$ Cyg, $\pi$ Dra, 46 Dra, $\gamma$ Equ, 112 Her, $\phi$ Her, $v$ Her, $\theta$ Leo, $\mu$ Lep,

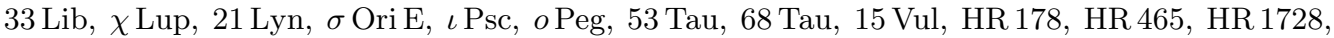
HR 1890, HR 4072, HR 7143, HR 7245, HR 7361, HR 7664, HR 7775, HR 8349, HD 965, HD 29647, HD 101065, HD 166473, HD 213637, HD 215441, HD 217522)

\section{The classes of CP Stars-visual spectroscopy}

Spectroscopic anomalies among B- and A-stars were known before astronomers made the distinction between upper and lower Main Sequences. The early history of this work has been amply reviewed (Bidelman 1967, Wolff 1983). Preston (1974) introduced the designation CP for chemically peculiar stars on the upper Main Sequence. His CP1 stars were previously called Am or Fm. The magnetic CP2 stars had anomalous lines of Sr, $\mathrm{Cr}, \mathrm{Eu}$, and $\mathrm{Si}$, which could be seen on classification $\left(125 \AA \mathrm{mm}^{-1}\right)$ spectra. CP3 stars were typically late B objects. Their detection was difficult at classification resolution. The extent of their peculiarities became increasingly apparent only on higher dispersion spectra. They have anomalously strong manganese lines and usually also $\lambda 3984$ of $\mathrm{Hg}$ II, and are commonly called HgMn stars.

Jaschek \& Jaschek (1974) describe the various classes of CP stars and introduce the notion of "visual spectroscopy." This can be done at both high and low dispersion. Workers 
who used high dispersion, like the Jascheks themselves, Bidelman (1966), or Cowley and coworkers (cf. Cowley 1976) made qualitative inferences about the abundances from the characteristics of the spectra. While this procedure lacked the rigor of a fine analysis, it was closely connected to the observations, and therefore not subject to the uncertainties of atomic parameters or poor determinations of effective temperature. Visual spectroscopy was often more valuable than ostensibly more rigorous analyses. We eschew an invidious listing of specific examples, but there are some interesting cases for which this is true.

Modern abundance work, especially supplemented by the atomic data bases made available by R. L. Kurucz (cf. Kurucz 2004), VALD (Kupka et al. 1999), DREAM (cf. Biemont 2004), etc., is less likely to go astray. Modern workers have now produced a rather large body of abundances of CP stars. We propose they be classified and analyzed by multivariate techniques, which should become standard tools. They will provide powerful supplements to the traditional classification methods.

\section{Cluster analysis}

This method uses the individual abundances as points in an $n$-dimensional space, where $n$ is the number of abundances. Initially, if $N$ stars are considered, there are $N$ clusters. Various algorithms are used to then merge the clusters into a smaller number of objects, which may be used as a basis of classification. We have employed a simple method that finds the closest pair of points, and merges them into a single cluster with a position that is the average of the two points. The next closest points are then found, and similarly merged. We have used Euclidian distances, though other choices are possible. The progress of the merging is illustrated on a tree or dendrogram, where the vertical

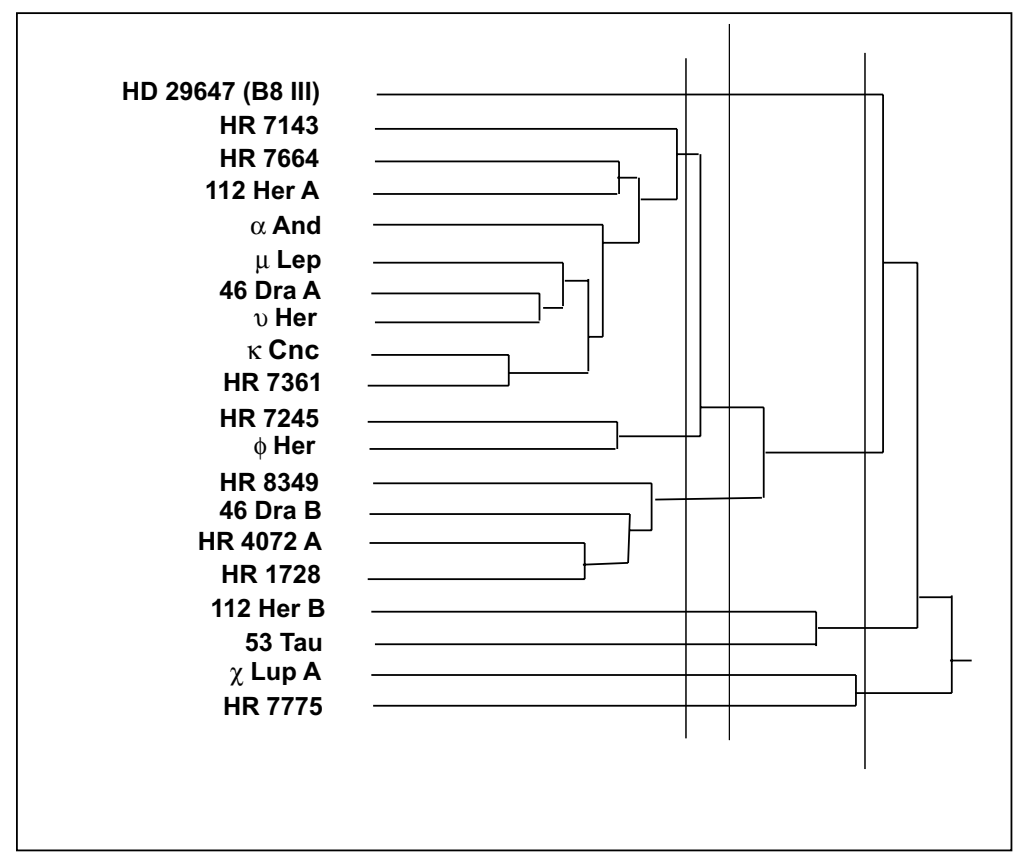

Figure 1. Dendrogram for CP stars. Abundances from Adelman, Castelli, Ryabchikova, Wahlgren, and coworkers. See text. 


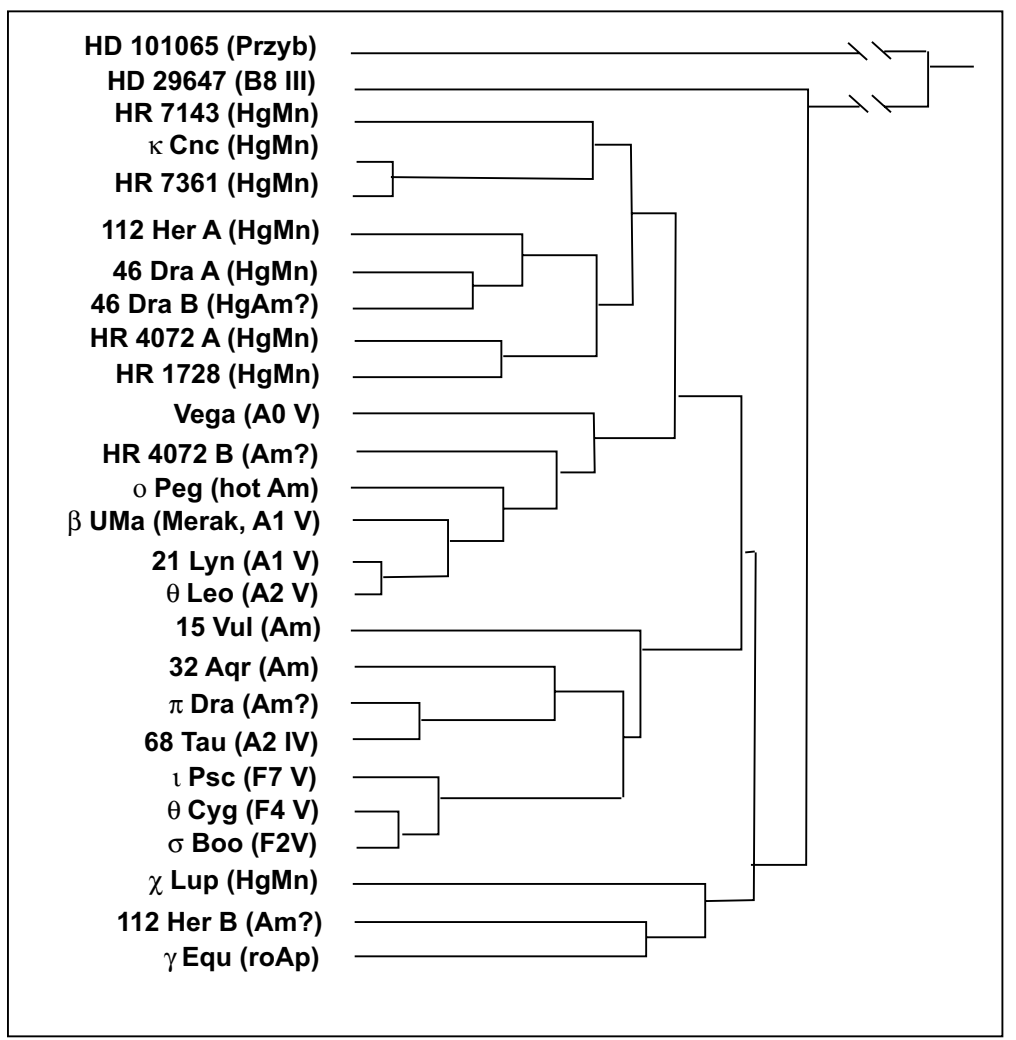

Figure 2. Dendrogram for CP stars. Abundances from Adelman, Castelli, Ryabchikova, Wahlgren, and coworkers. See text.

dimension shows the clusters. The horizontal dimension is a measure of the distance of the clusters from one another.

In most multivariate studies, some decision must be made to allow for missing data. There is no unique choice of how to deal with this. In the provisional studies below, we have filled in missing abundances with "solar" values. One must keep in mind that this can bias the proximity of stars in their multidimensional space.

\subsection{HgMn stars}

Fig. 1 shows a dendrogram for selected HgMn stars. The vertical cuts show possible means of separating the objects into classes. Cuts further to the right result in fewer classes. References to the data for individual stars in this and the following figure that are not cited elsewhere in the text are: Adelman (1994), Adelman et al. (1998, 2001), Ryabchikova et al. (1997, 1999), Leckrone et al. (1999), and Wahlgren et al. (2000).

\section{2. $C P 1, C P 2$, and $C P 3$ objects}

In Fig. 2, we apply the cluster method to a more heterogeneous group of stars. Those familiar with the individual spectra will not be surprised by the groupings, in most cases. The pairing of 112 Her B with $\gamma$ Equ may seem odd. The brighter star of the SB2, 112 Her A, is a well-known HgMn star, while $\gamma$ Equ is a cool roAp star (see Kurtz 1990, 2003 for a full description of the properties of the roAp stars). However, Ryabchikova et al. (1996) have already noted the similarity of 112 Her B to Am and roAp stars. 
Table 1. Correlation matrix for selected elements based on Erspamer-North abundances.

\begin{tabular}{lcccc}
\hline & $\mathrm{Ca}$ & $\mathrm{Sc}$ & $\mathrm{Ti}$ & $\mathrm{V}$ \\
\hline $\mathrm{Sc}$ & 0.54 & 1.00 & & \\
$\mathrm{Ti}$ & 0.65 & 0.26 & 1.00 & \\
$\mathrm{~V}$ & 0.09 & -0.08 & 0.41 & 1.00 \\
$\mathrm{Cr}$ & 0.47 & 0.03 & 0.82 & 0.48 \\
$\mathrm{Mn}$ & 0.49 & 0.23 & 0.62 & 0.37 \\
$\mathrm{Fe}$ & 0.57 & 0.05 & 0.85 & 0.47 \\
$\mathrm{Co}$ & 0.05 & 0.01 & 0.09 & 0.12 \\
$\mathrm{Ni}$ & 0.34 & -0.07 & 0.67 & 0.51 \\
$\mathrm{Sr}$ & 0.17 & -0.25 & 0.51 & 0.44 \\
$\mathrm{Y}$ & 0.20 & 0.07 & 0.54 & 0.49 \\
$\mathrm{Zr}$ & 0.06 & -0.26 & 0.27 & 0.38 \\
$\mathrm{Ba}$ & 0.06 & -0.25 & 0.48 & 0.51 \\
$\mathrm{Ce}$ & -0.17 & -0.39 & 0.06 & 0.26 \\
\hline
\end{tabular}

\subsection{Automated abundances of $A$ and F stars}

We performed cluster analysis and made correlation matrices using the results of Erspamer \& North's (2003) automated abundance calculations for 140 stars. The full dendrogram is too large to display here. It is available on the web at

$$
\text { http://www.astro.lsa.umich.edu/users/cowley/tree.htm }
$$

Likewise, the full correlation matrix may be seen at

http://www.astro.lsa.umich.edu/users/cowley/table6.htm

A portion is shown in Table 1.

The formal significance of a correlation coefficient depends on the number of points used in its calculation. However, to actually judge the reality of a correlation, one should exclude the default, solar values. In Figs. 3 and 4, stars with such default abundances are not plotted.

Erspamer-North AA, 398

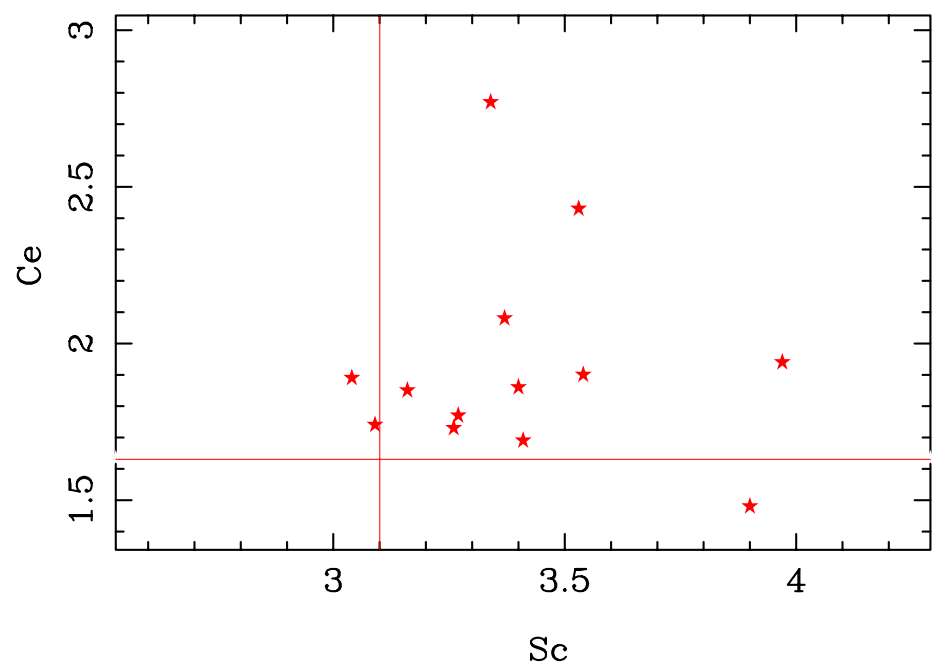

Figure 3. Cerium abundances plotted vs. those for scandium, showing a marginal anti-correlation at best. Vertical and horizontal lines show the solar abundance of Sc and Ce, respectively. Data from Erspamer \& North (2003). 
Erspamer-North AA, 398

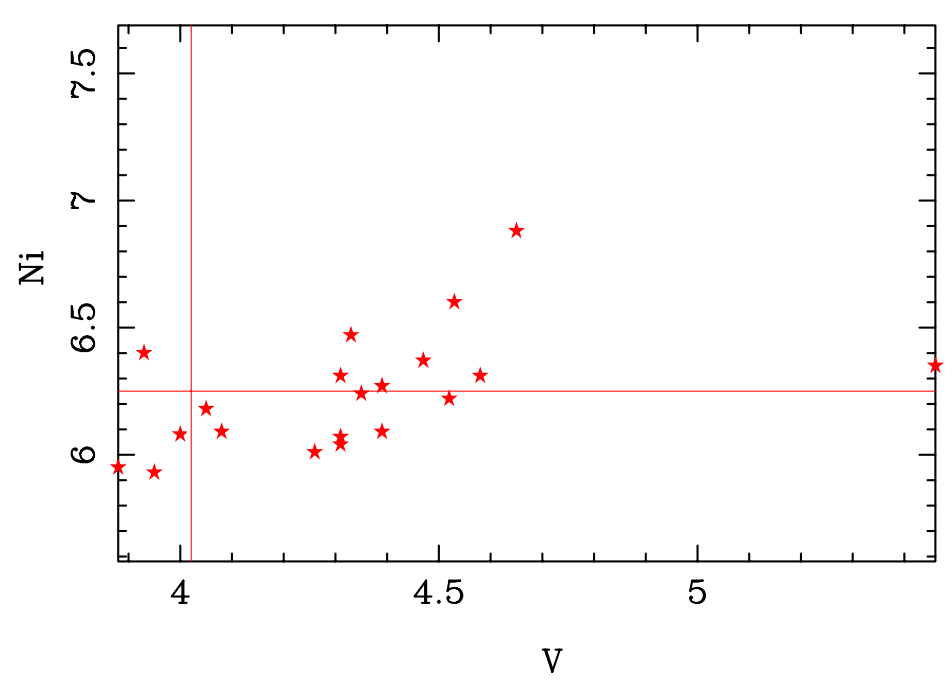

Figure 4. Ni abundances plotted vs. those for vanadium, showing a good correlation. Data from Erspamer \& North (2003).

\section{AA or AA: anomalous abundances or anomalous atmospheres}

IAU Symposium 26 was on Abundance Determinations in Stellar Spectra. In it, Anne Underhill (1966) gave a critique of analytical methods, and ended with the controversial conclusion that many of the traditional abundance anomalies might instead be the result of anomalous atmospheric structure. At that time, most abundances were determined using highly schematic model stellar photospheres. The slab, or Schuster-Schwarzschild model assumed single, characteristic values of the temperature and pressure. MilneEddington models allowed some depth dependence, but assumed constant ratios of the line to continuous absorption coefficient. Differential methods were widely used, where a single set of parameters, temperature, pressure, and surface gravity, was assumed to apply to each of two stars. The methods are described in classical textbooks.

Depth-dependent numerical models were just coming into their own, and it seemed possible that some variations from the classical temperature-pressure structure might give rise to effects that could be interpreted as abundance anomalies.

Abundance determinations at that time rested on the assumption of local thermodynamic equilibrium (LTE). This assumption was generally consistent with observations of stellar spectra. However, it was in principle subject to confirmation by a more fundamental non-LTE approach, which was practically beyond the techniques of the 1960s. Many astronomers wondered about the extent to which a rigorous non-LTE treatment would remove abundance anomalies in stars. Underhill put the question trenchantly: Do we have AA (anomalous abundances) or AA (anomalous atmospheres). In this regard, it should be noted that in the first half of the 20th century, it was widely believed that almost all stars had the same abundance distribution as the Sun. A. Unsöld (1955), the doyen of analytical stellar spectroscopy, wrote in his classical text that "it therefore seems justified to speak with caution of a cosmic abundance distribution of the elements." [tr. $\mathrm{CRC}$. A few obvious discordant cases, such as the R, N, and S stars were apparently set aside. 
Detailed LTE-modelling and realistic non-LTE calculations have mostly confirmed the abundance peculiarities of a wide variety of stars, including the CP stars (cf. Van't VeerMenerret 1963, Conti 1965, and various papers in Piskunov et al. 2003). Yet it is now clear that the spectra of many of these stars cannot be described by classical, plane-parallel, chemically homogeneous model atmospheres.

Within a decade of IAU Symposium 26, it was generally accepted that CP2 stars had horizontal abundance variations, chemically distinct continents. Stellar rotation, when combined with the surface abundance variations could account for the spectral, color, and light variations of these stars.

Nevertheless, it was thought the atmospheres were reasonably well described by classical models, at least within an abundance patch. Spectra were still analyzed ignoring complications due to horizontal abundance variations, which would essentially render the spectra composite. These analyses were reasonably consistent, insofar as the effective temperatures of the models were in general agreement with the degrees of excitation and ionization determined from various elemental species. This remained true for the observational and analytical tools available for much of the latter half of the 20th century.

Recently, it has become clear that classical models do not describe the vertical structure of at least CP2 stars, even within a putative abundance patch. The rapidly-oscillating or roAp stars are good examples. The cores of their low-Balmer members show anomalous structure (Cowley et al. 2001), and anomalous ionization is found for several of the lanthanides (Ryabchikova et al. 2004). The catchword, used through much of this symposium, is "stratification."

There is nothing new about a chemically stratified atmosphere. It has been known for years that the Earth's upper atmosphere, above about $100 \mathrm{~km}$, is chemically stratified, according to the molecular weights of atoms, ions, and molecules. Numerous studies (cf. Feldman \& Widing 2003) have shown that the solar atmosphere is vertically stratified. Elements with first ionization potentials below about $10 \mathrm{eV}$ are some 2 to 4 times more abundant in the chromosphere and corona than in the photosphere.

What is new is that calculations are now being made of CP star spectra, based on models with explicit elemental stratification. These models have achieved a modicum of success. For example, Kochukhov et al. (2003) have shown how the core-wing anomaly of cool CP2 stars could be accounted for in terms of a localized hot region in the high photospheres. Likewise, ionization and excitation anomalies may be accounted for in terms of chemical stratification (cf. Ryabchikova et al. 2004, and contributions in this Symposium).

\section{Abundance patterns}

The history of matter is written into its abundance patterns. We measure abundances today in stars, nebulae, and planets, and try to discern the processes that yielded them. Abundance patterns arise from nuclear and non-nuclear (e.g., chemical) processes. To some extent, each leave characteristic signatures (cf. Guthrie 1971). Nuclear processes are readily recognized. Well before the nature of atoms and nuclei were understood, the remarkable William Harkins (1917) wrote of "...a relation between the abundance of the elements and the structure of the nuclei of atoms." His work presaged the later and more influential compilation of Suess \& Urey (1956) of elemental abundances. These, and subsequent refinements were called, as we have noted above, cosmic abundances. Audouze \& Tinsley (1976) suggested the more useful and accurate term standard abundance distribution, or SAD. It is shown in Fig. 5. 
Logarithmic SAD Abundances: $\log (\mathrm{H})=12.0$

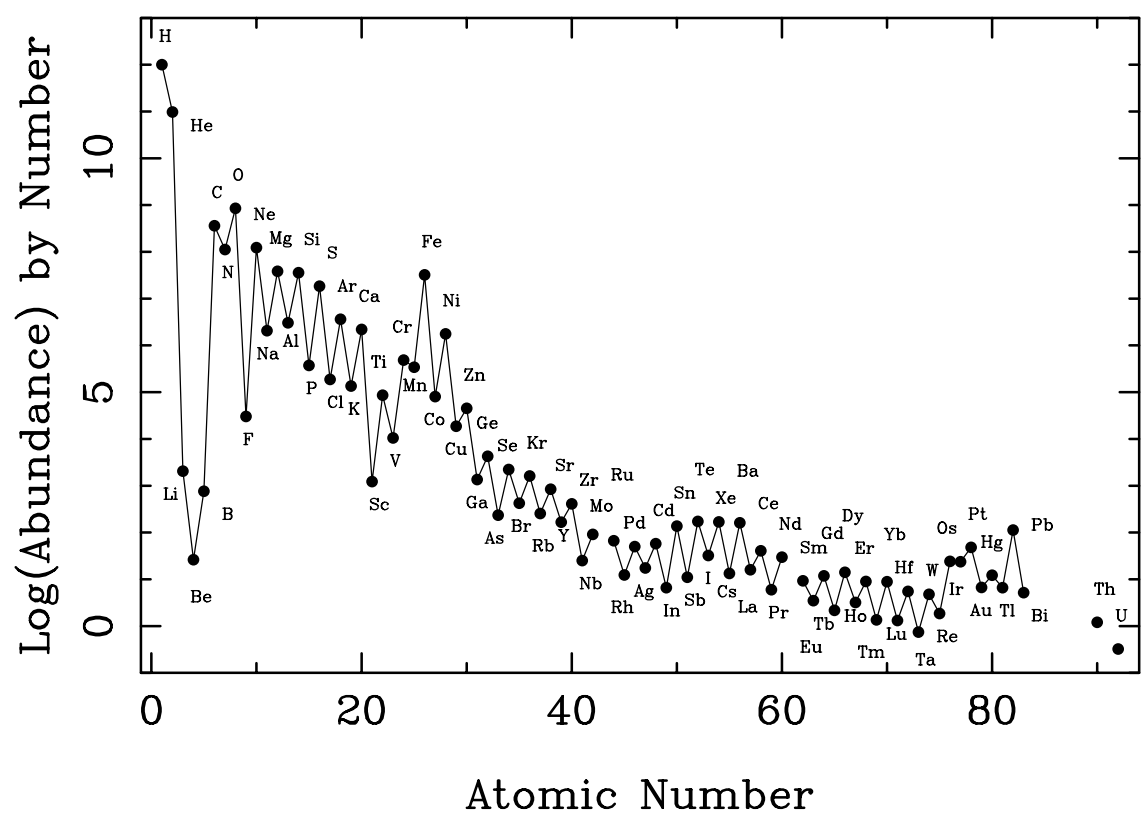

Figure 5. The standard sbundance sistribution (SAD), formerly known as the "cosmical abundance distribution;" data are from Grevesse \& Sauval (1998).

Logarithmic Crustal Abundances: $\log (\mathrm{Si})=6.0$

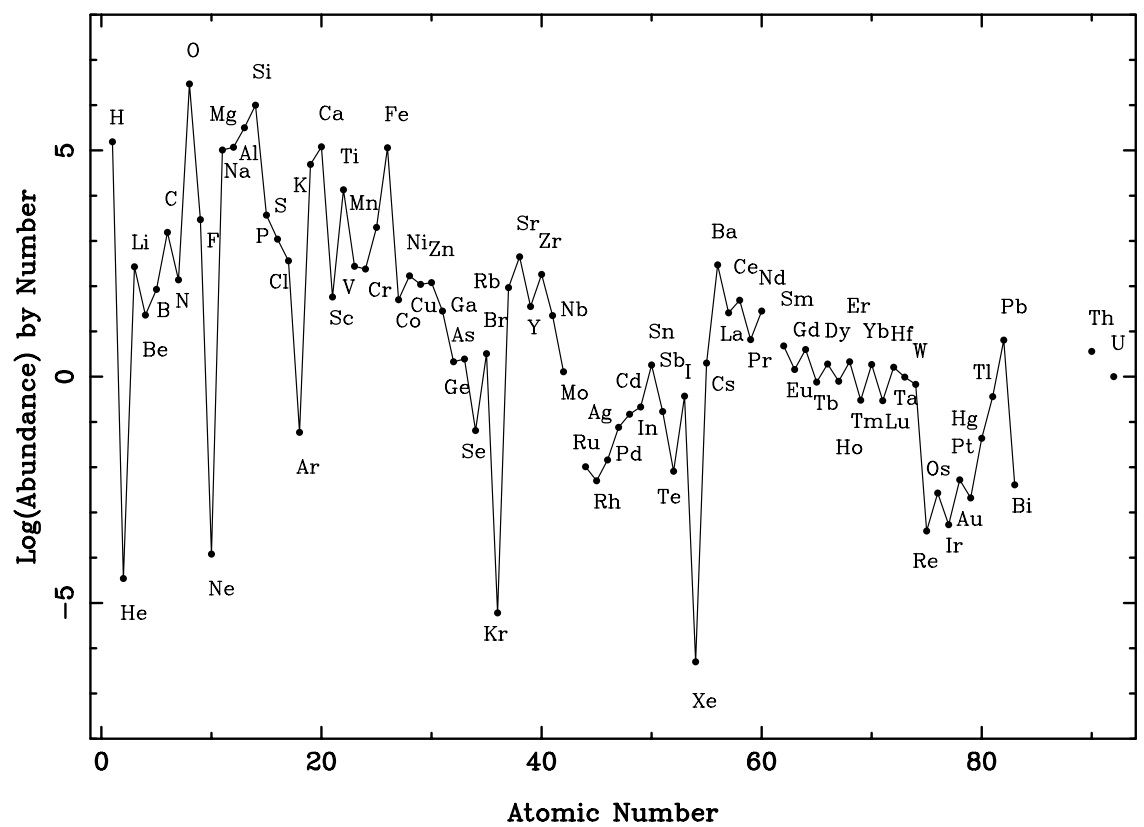

Figure 6. Abundances in the Earth's crust. The result of differentiation from the SAD. Data are from Mason (1966). 


\section{Sun and HR 7143}

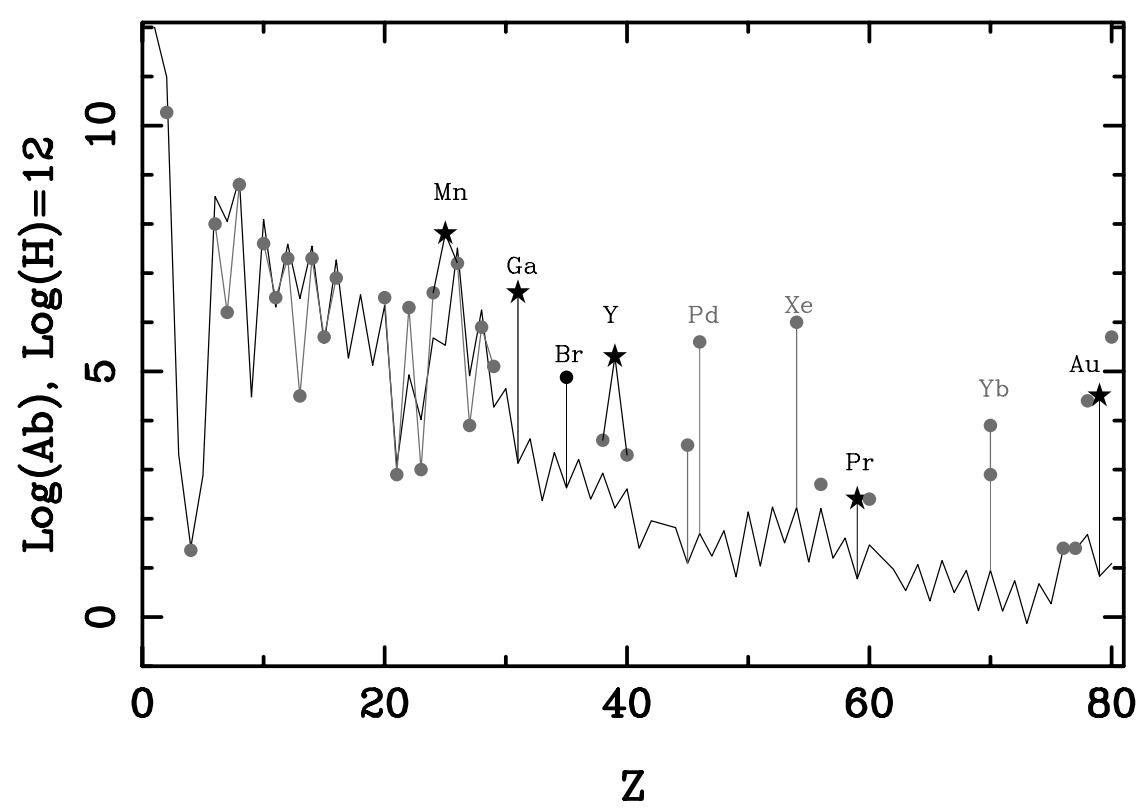

Figure 7. SAD abundances along with those HR 7143 (HD 175640) from Castelli \& Hubrig (2004). Vertical lines are drawn to help indicate whether the points belong to an odd- or even-Z element. Here, and in the two following figures, filled circles and stars indicate the stellar abundances; stars are used to indicate variations from a nuclear pattern. The two points for $\mathrm{Yb}$ come from $\mathrm{YbII}$ and $\mathrm{Yb}$ III lines.

Standard astronomical practice is to concentrate on departures from the SAD. We note that plots of $[\mathrm{El} / \mathrm{H}]$ vs. Z obscure one of the most notable and basic characteristics of nuclear patterns, namely the odd-even alternation. There are certainly times when it is desirable to suppress this pattern. In the following figures, we plot logarithms of the stellar abundances directly, and not their ratio to the SAD.

Fig. 6 shows a decidedly non-nuclear pattern, that of the Earth's crust. Note the huge even- $\mathrm{Z}$ anomalies that occur at the noble gasses. These and other characteristics of the crustal abundance pattern are well understood in terms of the thermodynamics of Earth materials.

\section{CP star abundance patterns}

\section{1. $H R 7143=H D 175640$}

The CP stars that show the most obvious departures from a nuclear abundance pattern are the CP3, or Mercury-Manganese stars. Given that the SAD abundance of manganese is roughly 2 dex less than that of iron, a star can show a substantial manganese excess without violating the expectations of nuclear processing in the region of the iron peak. For example, Cowley \& Aikman (1975) showed that quasi-equilibrium calculations could lead to a $\mathrm{Mn}(\mathrm{Z}=25)$ abundance greater than that of $\mathrm{Cr}(\mathrm{Z}=24)$, with $\mathrm{Fe}(\mathrm{Z}=26)$ only a few tenths of a dex greater than that of Mn. However, in $53 \mathrm{Tau}, \mathrm{Mn}$ is surely more abundant than either $\mathrm{Cr}$ or Fe, severely violating an expected nuclear pattern (Smith \& Dworetsky 1993). Oddly, the star 53 Tau shows no $\lambda 3984$ Hg II line. 
Sun and 32 Aqr

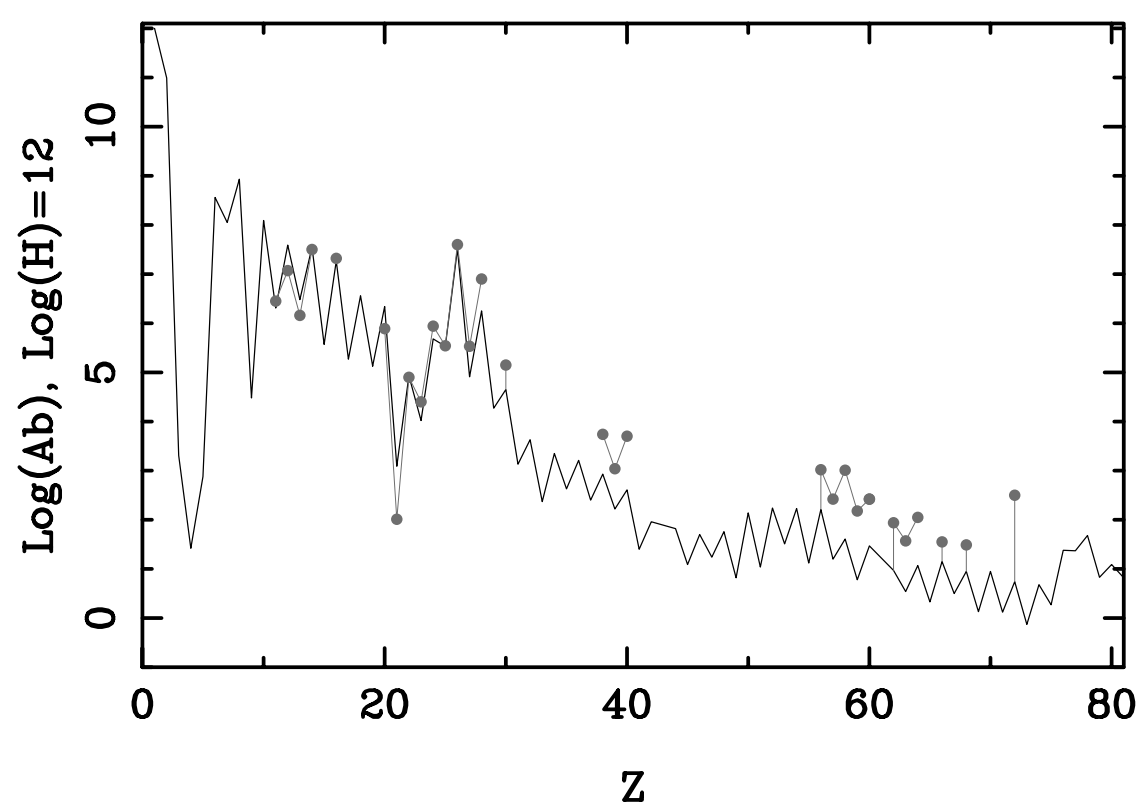

Figure 8. SAD abundances along with those of a quintessential Am star, 32 Aqr (HR 8410, HD 209625). Abundances from Adelman et al. (1997) show no obvious odd- or even-Z anomalies. See caption to Fig. 7

Castelli \& Hubrig (2004) found that the HgMn star HR 7143 (HD 175640) also has more $\mathrm{Mn}$ than either $\mathrm{Cr}$ or Fe. Fig. 7 is based on their paper. The odd-Z anomalies at $\mathrm{Mn}, \mathrm{Ga}, \mathrm{Br}, \mathrm{Y}, \mathrm{Pr}$, and $\mathrm{Au}$ are indicated. The abundance patterns in the HgMn stars provide the most obvious signs of non-nuclear processes.

\subsection{Aqr}

We have considered 32 Aqr (HR 8410) to be a quintessential metallic-lined (Am) star. HR 178 and 63 Tau, which have been studied extensively by Van't Veer-Menneret and her colleagues (cf. Van't Veer-Menneret et al. 1988, Van't Veer-Menneret 1963), are similar Am stars with relatively sharp spectral lines. The Am (or CP1) stars are not identical, but their compositions are more uniform than those of the magnetic (CP2) sequence.

Michaud (2004) and his coworkers (cf. Richer et al. 2000) have made detailed diffusion calculations for stars in the mass range appropriate to the Am and cool CP2 stars. These calculations provide a basis for understanding the lower abundances of the CNO elements, which is generally characteristic of CP stars. Like previous diffusion calculations, they tend to predict abundance enhancements of elements with naturally low abundances, and thus a kind of inverse odd-even effect. While such patterns are realized among the HgMn stars, there is little or no indication of them in the Am stars. This is illustrated in Fig. 8.

\section{3. $H D 213637$}

Kochukhov (2003) determined abundances for 36 elements in the remarkably cool $\left(\mathrm{T}_{\text {eff }}\right.$ $=6400 \mathrm{~K}$ ) roAp star HD 213637. They are displayed graphically in Fig 9. Abundances for several of the lanthanides have two values, with the higher value coming from the 


\section{Sun and HD 213637}

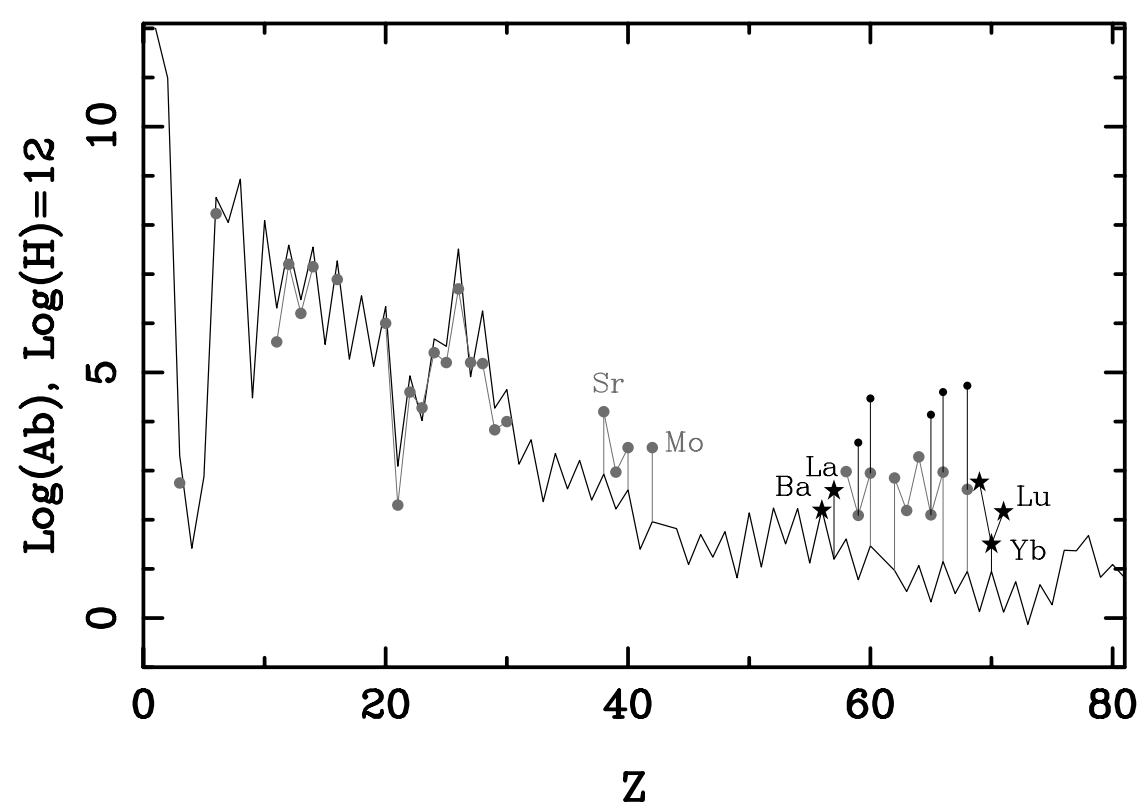

Figure 9. SAD abundances along with those of the cool roAp star HD 213637. Abundances from Kochukhov (2003). See caption to Fig. 7

third spectra. A possible even- $\mathrm{Z}$ anomaly is seen at $\mathrm{Z}=70$, where $\mathrm{Yb}$ is less abundant than its odd-Z neighbors. This must be confirmed with better oscillator strengths and a definitive model. Kochukhov finds different abundances from weak and strong lines of numerous species, as well as from high- and low-excitation lines. This is attributed to stratification of the elemental abundances.

The point for $\mathrm{Ba}(\mathrm{Z}=56)$ could also be an even- $\mathrm{Z}$ anomaly, though no abundance is available for $\mathrm{Cs}(\mathrm{Z}=55)$. The $\mathrm{Ba}$ abundance varies considerably among the CP2 stars, unlike that of $\mathrm{Sr}(\mathrm{Z}=38)$, which is typically enhanced.

\section{High-energy phenomena associated with CP Stars}

\subsection{Radio observations}

Drake (1998) reviewed the subject of radio continuum observations of magnetic CP stars. We will briefly summarize the principal conclusions from his review, and then touch on developments that have occurred since its publication. We will also consider the status of radio observations of the non-magnetic CP stars.

Examination of magnetic CP stars in the radio regime began in the 1970s at centimeter wavelengths, but failed to produce any evidence for strong radio emission from this class of objects. Out of some two dozen stars surveyed, only Babcock's star (HD 215441) showed a weak 50 mJy $(2 \sigma)$ radio excess (Kodaira \& Fomalont 1970). By the end of the 1980s, with the advent of the VLA and similar arrays, five magnetic CP stars (out of 50 investigated) were identified as radio sources, including three He-strong stars and two Si stars (cf. Drake et al. 1985, 1987). No later-type SrCrEu magnetic CP stars were detected in these early surveys. Phillips \& Lestrade (1988) demonstrated that the radio emission from the high luminosity He-strong stars was nonthermal in origin. 
Leone (1991) and Leone \& Umana (1993) discovered that the radio emission from $\sigma$ Ori E (HD 37479) and HR 1890 (HD 37017), two of the first He-strong radio sources identified, varied with the phase of the magnetic field as the stars rotated. The observed modulation suggested that the radio emission arose from a stable co-rotating magnetosphere. Linsky et al. (1992) reported detections of three out of nine He-strong stars and 13 out of 38 He-weak/Si-type stars in the Orion OB1 and Sco OB2 associations, but no detections of cooler Ap stars out of 14 sampled. These authors also developed a model for producing the radio radiation involving gyrosynchrotron emission by mildly relativistic, nonthermal electrons in optically thick tori near the magnetic equators of these stars.

Further work by Leone et al. (1994) and Drake and his collaborators (cf. Drake 1998) raised the total of radio emitting He- and Si-type CP stars to nearly three dozen. Correlations between the radio luminosity at the source, and fundamental stellar parameters for members of this group led Drake (1998) to the following empirical relation, accurate to \pm 0.6 dex:

$$
\log \left(L_{R}\right) \approx 15.1+\left(6.85 \cdot \log \left(T_{\text {eff }} / 10^{4}\right)\right)+\left(1.20 \cdot \log \left(H_{\mathrm{S} / \mathrm{kG}}\right)\right)-\left(0.60 \cdot \log \left(P_{\text {rot }} / \text { days }\right)\right) .
$$

Here, $L_{R}$ in in $\mathrm{ergs}^{-1} \mathrm{~Hz}^{-1}$ for observations at $6 \mathrm{~cm}$.

Recent work pertaining to the magnetic CP stars as radio sources has focused on confirming and expanding previous models to explain the radio emission. For example, Trigilio et al. (2004) have developed a 3D model in which they substantiate quantitatively the qualitative models proposed by Linsky et al. (1992) and others by reproducing the observed modulation in the $2 \mathrm{~cm}$ light curves for $\sigma$ Ori E and HR 1890. These authors emphasize the need for multi-frequency radio light curves to further test and/or constrain their model. Such improvements not withstanding, the number of bona fide radio emitting magnetic CP stars is substantial, the mechanism that produces such radiation appears to be largely understood, and there exists a means of predicting, to within reasonable limits, the radio luminosity of magnetic CP stars on the basis of their fundamental properties.

Searches for radio emission from non-magnetic CP stars (Am and HgMn stars) using the VLA by Drake et al. (1994) have proved fruitless. Twenty-three stars (11 Am's and 12 HgMn's) were surveyed at $3.6 \mathrm{~cm}$ for nonthermal radio flux and none were found to emit with an upper limit of $\leqslant 0.20 \mathrm{mJy}$. This result corroborated earlier efforts to detect radio emission from Am and HgMn stars conducted by Linsky et al. (1992) and White et al. (1993). The non-detection of these stars at radio wavelengths may not be too surprising if they follow an empirical relation like that quoted above, given their relatively cool temperatures and weak magnetic fields.

\subsection{X-ray observations}

Among the chemically peculiar stars, the magnetic CP stars (CP2s) have provided obvious targets for observation in the X-ray regime going back to the era of the Einstein and EXOSAT missions. Drake et al. (1987) discovered two X-ray sources, both early type He-strong stars, with emission rates comparable to those of hot, non-magnetic OB stars and concluded that the emitting regions in these sources are related to stellar winds and not to the presence of magnetospheres. The launch of ROSAT in 1990 provided detection sensitivities 10 to 100 times greater than previous X-ray observatories, and studies of the ROSAT All-Sky Survey by Drake et al. (1994) yielded ten detections out of 100 magnetic CP star positions examined. Five of the sources possessed properties indicating that the X-rays originated from a stellar wind-type mechanism and were unrelated to the magnetic peculiarities of the host star. Of the remaining five sources, Drake and coworkers concluded that two He-weak and two Si stars may represent intrinsic X-ray sources whose emission is of magnetospheric origin. Their final source, the $\mathrm{SrCrEu}$ star $52 \mathrm{Her}$, 
is a triple star system wherein the the X-ray emission can plausibly be associated with one of the other cooler components. A similarly low detection rate for magnetic CP stars as X-ray emitters was found by Leone (1994) in his re-examination of Einstein images: in a sample of 90 sources associated with B-type stars, only four were known magnetic CP stars, and of those, three were identified as binary systems.

Drake (1998) has extended the program of Drake et al. (1994) to include archival, pointed ROSAT data and has culled out an additional 18 magnetic CP star candidates with intrinsic X-ray emission. Included in this group is Babcock's star, as well as another 13 He-weak and Si stars, plus four SrCREu-type objects. Despite the increase in potential X-ray emitting CP stars, Drake finds no correlation between the X-ray emission characteristics of these stars and any other of their known physical properties. This is in striking contrast to the radio emission from magnetic CP stars which is predictable from their magnetic field strength, temperature and rotation. Drake concludes that the case for X-ray emission from CP2 stars via magnetospheric activity (as opposed to stellar wind-induced effects) has yet to be persuasively made.

Observations of galactic clusters and associations have also revealed some CP2 stars as potential X-ray emitters. ROSAT investigations of the young open cluster NGC 2516 were made by Dachs \& Hummel (1996) and by Jeffries et al. (1997) that identified 4 to 6 magnetic CP cluster stars as X-ray sources. Jeffries and coworkers concluded that the emission was most probably intrinsic to the CP stars, although contributions from an unseen companion in each case could not be ruled out. Chandra X-ray observations of NGC 2516 by Damiani et al. (2003) have yielded a detection rate for dwarf A stars as X-ray emitters of only about $21 \%$ (12 out of 58 ) as a whole, but fully one-half of those discovered are chemically peculiar. Within the class of CP stars alone, the detection fraction is very high: six or seven out of eight. The observed X-ray luminosity function for the A star detections is compatible with those found for later-type stars, however, indicating that the X-ray emission could be associated with lower-mass companions.

Padovani (2005), using the extensive data handling and analysis capabilities of the Virtual Observatory, has found that CP A-type stars are 3 to 4 times more likely to be associated with X-ray sources than normal A-type stars, but that the detection rate for the magnetic CP A-types is comparable to that of all CP A-type stars. He concludes that the presence of a magnetic field does not play a significant role in triggering X-ray emission in this class of star. This result, together with characteristic X-ray luminosity function for the A-star detections, tends to corroborate Drake's (1998) assessment of the situation regarding the CP2 stars as intrinsic X-ray emitters.

The roAp stars may hold special promise for elucidating the potential for intrinsic $\mathrm{X}$-ray emission within the CP2 class. In addition to the clear presence of a magnetic field (see, e.g., Mathys et al. 1997 and Hubrig et al. 2004), these stars seem to possess shallow convection zones that are instrumental in exciting their pulsation. Moreover, none of the 32 or so known roAp stars has been proven to be a member of a binary system (Hubrig et al. 2000), so that any detected X-ray emission is likely to be associated with the magnetic star itself. Stelzer (2004, private communication) and colleagues have proposed to make Chandra observations of five roAp stars in an effort to address this and related questions.

Among the non-magnetic HgMn (or CP3) stars, eleven were detected as soft X-ray emitters in the ROSAT All-Sky Survey by Berghöfer et al. (1996). Hubrig et al. (1998) carefully examined each of these systems using all available data and concluded that in every case, the X-ray emission had a natural explanation in terms of a companion star: for seven stars, the X-ray emitter was likely a late-type main sequence star, while 
in four instances, a pre-main sequence object was required. Thus, like their cooler CP2 congeners, the HgMn stars do not appear to be intrinsic X-ray emitters.

\subsection{Surface nuclear reactions in Ap stars}

Recently, Cowley et al. (2004) have reported the identification of promethium in the spectra of HD 101065 and HD 965. Like an earlier report of Pm in the spectrum of HR 465 (Aller \& Cowley 1970), this one again raises questions about the possible source(s) of this short-lived $\left(T_{1 / 2} \leqslant 17.7 \mathrm{yrs}\right)$ radioactive element. Conventional theories of nucleosynthesis fail to provide plausible mechanisms for the existence of observable amounts of $\mathrm{Pm}$ in the atmospheres of unevolved stars. This has led to the consideration of nuclear reactions occuring on the surfaces of Ap stars as a means of producing measurable quantities of this species. Although scenarios involving such reactions are generally believed to require larger energy inputs than are sustainable by typical stars to work (cf. Tjin A Djie et al. 1973), Cowley (1973) demonstrated that some models, for example, ones involving $\mathrm{Nd}(\mathrm{p}, \mathrm{n}) \mathrm{Pm}$ reactions, could be made viable, assuming $\mathrm{Pm} / \mathrm{Nd}$ abundance ratios of $10^{-2}$ and $10 \mathrm{MeV}$ protons with energy flux densities of only $\approx 5 \cdot 10^{9} \mathrm{erg} \mathrm{cm}^{-2} \mathrm{~s}^{-1}$ over times of the order of 10 years. Padovani's (2005) discovery that Ap stars are 3 to 4 times more likely to be associated with X-ray sources than normal A stars suggests that high energy events commonly occur in the vicinity of (if not on) many Ap stars. Such events may provide the necessary flux of high energy particles (p's, n's, and $\alpha$ 's) to initiate nuclear reactions in their atmospheres. (In studies of 19 solar flares, Ramaty et al. (1995) found that the energy associated with expelled particles equalled or exceeded that of the nonrelativistic electrons that produce the hard X-rays in these events.) Similar arguments have been made for the production of $\mathrm{Li}$ in low mass secondaries by CNO spallation reactions caused by high energy protons generated in transient X-ray events from the compact object in these systems (Martín et al. 1994a,b).

In the light of this possibility, we review some recent results from solar and stellar flare studies. To provide order-of-magnitude estimates, we will assume the Ap star to be part of an X-ray emitting binary with a 10-day period. The value adopted for the period is a compromise choice: periods for CP3 binaries are generally less than about 5 days, while most binaries containing CP2 stars have periods greater than 10 days (Preston 1974). Although we will also focus our attention on proton-induced reactions only, it must be said that other production reactions exist.

Neutron addition to stable Nd isotopes to produce Nd-147 and/or Nd-149 that then beta decay to $\mathrm{Pm}$ is a possible production mechanism for this exotic species. However, the particle densities in the atmospheres of low mass X-ray stars are sufficiently high to effectively thermalize the neutrons emitted in the high energy events. In this case, the neutrons are consumed primarily in reactions producing deuterium in $H(n, \gamma) D$ reactions or tritium according to ${ }^{3} \mathrm{He}(\mathrm{n}, \mathrm{p}) \mathrm{T}$ (Hua \& Lingenfelter 1987), resulting in escaped neutron fluxes that are several orders of magnitude smaller than those of the protons. As Audouze (1970) remarks: "The action of thermal neutrons is negligible in the alteration of abundances of elements heavier than helium."

Promethium production by $\alpha$-addition on Pr-141 (the only stable isotope of the element) to yield Pm-143 is also a possibility. However, the $\alpha / \mathrm{p}$ ratio in typical Sun-like flares is only 0.1-0.5 for energies greater than about $1 \mathrm{MeV}$ per nucleon (MacKinnon \& Toner 2003). For particle energies in the range 10-50 MeV per nucleon, the smaller flux of $\alpha$ 's, coupled with the larger Coulomb barrier to $\alpha$ reactions, makes this scenario of less importance than one involving protons.

Leya et al. (2003) have modeled the production of short-lived nuclides by solar energetic particles, adopting a proton fluence of $10^{23} \mathrm{~cm}^{-2}$. Assuming a typical flare area 
of about $10^{17} \mathrm{~cm}^{2}$ (Paesold et al. 2003), the mean proton fluence over the solar surface is $1.6 \cdot 10^{17} \mathrm{~cm}^{-2}$. Ten large flares per year then yield an average proton flux of about $1.6 \cdot 10^{18} \mathrm{~cm}^{-2} \mathrm{yr}^{-1}$. For a CP binary with a 10-day period, the proton flux in the neighborhood of the Ap star will be $\approx 3 \cdot 10^{15} \mathrm{~cm}^{-2} \mathrm{yr}^{-1}$. Over ten years, the proton fluence will be $\approx 3 \cdot 10^{16} \mathrm{~cm}^{-2}$. It could be much larger if the protons were guided along magnetic field lines.

Following Audouze's (1970) methods for estimating the required proton fluence to produce heavy and rare earth elements by surface reactions, we adopt a mean inverse formation cross-section for production by $50 \mathrm{MeV}$ protons to be $10^{35}-10^{36} \mathrm{~cm}^{-2}$ (cf. Andouze's Table 3.). Taking the Nd-to-H ratio to be $3 \cdot 10^{-11}$ in the Sun (Grevesse \& Sauval 1998), we find a mean proton fluence for $\mathrm{Nd}$ formation in the Sun of 3 . $10^{24-25} \mathrm{~cm}^{-2}$. Assuming a Pm-to-Nd ratio of $10^{-2}$ and noting that Nd overabundances in Ap stars can reach $10^{3}$ or more relative to the Sun, we predict formation fluences for observable amounts of $\mathrm{Pm}$ to be in the range $3 \cdot 10^{23-24} \mathrm{~cm}^{-2}$. This is $10^{7-8}$ times larger than the 10-year proton fluence calculated above.

Proton fluences from flare activity on young stellar objects (YSOs) can be as much as $10^{5}$ times greater than on the Sun (Feigelson et al. 2002), so that if the companion to the Ap star were a YSO, then the available proton fluence might be as high as about $3 \cdot 10^{21}$ $\mathrm{cm}^{-2}$. Alternatively, if the magnetic fields associated with the flare regions were 1000 times greater than those on the Sun, then the expected fluence might be as much as a factor of $10^{6}$ larger or about $3 \cdot 10^{22} \mathrm{~cm}^{-2}$. In either of these cases, if the Nd abundance were to be a factor of 10 greater than assumed above (as it is in the case of HD 101065 [Cowley et al. 2000]), then it may just be marginally possible to produce measurable quantities of Pm in this manner.

From an evolutionary standpoint, it is difficult to reconcile the presence of a YSO in the company of a Main Sequence Ap star, although it has been shown that T Tauri stars possess multipolar magnetic fields like the Sun only with greatly enhanced strengths and surface coverage (Valenti \& Johns-Krull 2001) as needed to make heavy element production possible in the manner described above. Thus, the validity of this mechanism, even for stars as unusual as HD 101065, remains in question, and further work on this problem will be required to satisfactorily account for the characteristics of stars like HD 101065 .

\section{Acknowledgements}

We would like to acknowledge the useful comments from many colleagues. S. J. Adelman, W. P. Bidelman, and T. A. Ryabchikova have provided us with help and insights into the CP star puzzle for many years.

\section{References}

Adelman, S. J. 1994, MNRAS 266, 97.

Adelman, S. J., Ryabchikova, T. A. \& Davydova, E. S. 1998, MNRAS 297, 1.

Adelman, S. J., Caliskan, H., Kocer, D. \& Bolcal, C. 1997, MNRAS 288, 470.

Adelman, S. J., Snow, T. P., Wood, E. L., et al. 2001, MNRAS 328, 1144.

Aller, M. F. \& Cowley, C. R. 1970, ApJ 162, L145.

Audouze, J. 1970, A\&A 8, 436.

Audouze, J. \& Tinsley, B. M. 1976, ARA\& A 14, 43.

Bearman, P.W. \& Graham, J.M.R. 1980, J. Fluid Mech. 99, 225.

Berghöfer, T.W., Schmitt, J.H.M.M. \& Cassinelli, J.P. 1996, A $\& A S$ 118, 481.

Bidelman, W. P. 1966, in Abundance Determinations in Stellar Spectra, IAU Symp. 26 ed. H. Hubenet (New York: Academic Press) p. 118. 
Bidelman, W. P. 1967, in The Magnetic and Related Stars 1967, R. C. Cameron, ed. (Baltimore: Mono Book Corp.) p. 29.

Biémont, E. 2004, in 8th International Colloquium on Atomic Spectra and Oscillator Strengths for Astrophysical and Laboratory Plasmas, to appear in Phys. Scr., T Ser.

Castelli, F. \& Hubrig, S. 2004, A\&A in press.

Conti, P. S. 1965, ApJS 11, 47.

Cowley, C.R. 1973, The Observatory 93, 195.

Cowley, C. R. 1976, ApJS 32, 631.

Cowley, C. R. \& Aikman, G. C. L. 1975, ApJ 196, 521.

Cowley, C.R., Bidelman, W.P., Hubrig, S., et al. 2004, A\&A 419, 1087.

Cowley, C. R., Hubrig, S., Ryabchikova, T. A., et al. 2001, A\&A 367, 939,

Dachs, J. \& Hummel, W. 1996, A\&A 312, 818.

Damiani, F., Flaccomio, E., Micela, G., et al. , 2003 ApJ 588, 1009.

Drake, S.A. 1998, Contrib. Astron. Obs. Skalnaté Pleso 27, 382.

Drake, S.A., Abbott, D.C., Linsky, J.L., Bieging, J.H. \& Churchwell, E. 1985, in Radio Stars, eds. R.M. Hjellming \& D.M. Gibson (D. Reidel: Dordrecht), p. 247.

Drake, S.A., Abbott, D.A., Bastian, T.S., et al. , 1987, ApJ 322, 902.

Drake, S.A., Linsky, J.L., Schmitt, J.H.M.M. \& Rosso, C. 1994, ApJ 420, 387.

Drake, S.A., Linsky, J.L. \& Bookbinder, J.A. 1994, AJ 108, 2203.

Erspamer, D. \& North, P. 2003, A\&SA 398, 1121.

Feigelson, E.D., Garmire, G.P. \& Pravdo, S.H. 2002, ApJ 572, 335.

Feldman, U. \& Widing, K. G. 2003, Sp. Sci. Rev. 107, 665.

Grevesse, N. \& Sauval, A. J. 1998, Sp. Sci. Rev. 85, 161.

Guthrie, B. N. G. 1971, Ap\&SSS 13, 168.

Harkins, W. D. 1917, J. Am. Chem. Soc. 39, 856.

Hubrig, S., Berghöfer, T.W. \& Mathys, G. 1998, Contrib. Astron. Obs. Skalnaté Pleso 27, 464.

Hubrig, S., Kharchenko, N., Mathys, G. \& North, P. 2000, A\&A 3551031.

Hubrig, S., Kurtz, D.W., Bagnulo, S., et al. 2004, A\&A 415, 661.

Hua, X.-M. \& Lingenfelter, R.E. 1987, ApJ 319, 555.

Jaschek, M., and Jaschek, C. 1974, Vistas in Astron. 16, 131.

Jeffries, R.D., Thurston, M.R. \& Pye, J.P. 1997, MNRAS 287, 350.

Kodaira, K. \& Fomalout, E.B. 1970, ApJ 161, 1169.

Kochukhov, O. 2003, A\& A 404, 669.

Kochukhov, O., Bagnulo, S. \& Barklem, P. S. Modelling of Stellar Atmospheres, IAU Symp. 210, N. Piskunov, W. W. Weiss \& D. F. Gray, eds. (Provo Utah: ASP-CS \& IAU), D17.

Kupka, F., Piskunov, N., Ryabchikova, T. A., Stempels, H. C. \& Weiss, W. W. 1999, A\&AS $138,119$.

Kurtz, D.W. 1990, ARAEA 28, 607.

Kurtz, D.W. 2003, Ap\&SS 284, 29.

Kurucz, R. L. 2004, http://kurucz.harvard.edu.

Leckrone, D. S., Proffitt, C. R., Wahlgren, G. M. et al. 1999, AJ 117, 1454.

Leone, F. 1991, A\&A 252, 198.

Leone, F. 1994, A\&A 286, 486.

Leone, F. \& Umana, G. 1993, A\&A 268, 667.

Leone, F., Trigilio, C. \& Umana, G. 1994, A\&A 283, 908.

Leya, I., Halliday, A.N. \& Wieler, R. 2003, ApJ 594, 605.

Linsky, J.L., Drake, S.A. \& Bastian, S.A. 1992, ApJ 393, 341.

MacKinnon, A.L. \& Toner, M.P. 2003, A\& A 409, 745.

Martín, E.L., Spruit, H.C. \& van Paradijs, J. 1994a, A\&A 291, L43.

Martín, E.L., Rebolo, R., Casares, J. \& Charles, P.A. 1994b, ApJ 435, 791.

Mason, B. 1966, Principles of Geochemistry, 3rd. ed. (New York: John Wiley), Table 3.3.

Mathys, G., Hubrig, S., Landstreet, J.D., et al. 1997, A\&AS 123, 353.

Michaud, G. 2005, These Proceedings, 173

Padovani, P. 2005, These Proccedings, 485 
Paesold, G., Kallenbach, R. \& Benz, A.O. 2003, ApJ 582, 495.

Piskunov, N., Weiss, W. W. \& Gray, D. F., eds. 2003, Modelling of Stellar Atmospheres, IAU Symp. 210 (Provo Utah: ASP-CS \& IAU).

Phillips, R.B. \& Lestrade, J.-F. 1988, Nature 334, 3239.

Preston, G.W. 1974, ARA\&A 12, 257.

Ramaty, R., Mandzhavidze, N., Kozlovsky, B. \& Murphy, R.J. 1995, ApJ 455, L193.

Richer, J., Michaud, G. \& Turcotte, S. 2000, Ap. J. 529, 338.

Ryabchikova, T. A., Malanushenko, V. P. \& Adelman, S. J. 1999, A\&A 351, 963.

Ryabchikova, T. A., Zakharova, L. A. \& Adelman, S. J. 1996, MNRAS 283, 1115.

Ryabchikova, T. A., Adelman, S. J., Weiss, W. W. \& Kuschnig, R. 1997, A $\& A$ 322, 234.

Ryabchikova, T. A., Nesvacil, N., Weiss, W. W., Kochukhov, O. \& Stütz, Ch. 2004, A\&A 423, 705.

Smith, K. C. \& Dworetsky, M. M. 1993, A\&̛A 274, 335.

Suess, H. E. \& Urey, H. C., 1956, Rev. Mod. Phys. 28, 53

Tjin A Djie, H.R.E., Takens, R.J. \& van den Heuvel, E.P.J. 1973, Astrophys. Letters 13, 215.

Trigilio, C., Leto, P., Umana, G., Leone, F. \& Buemi, C.S. 2004, A\&A 418, 593.

Underhill, A. B. 1966, in Abundance Determinations in Stellar Spectra, IAU Symp. $26 \mathrm{H}$. Hubenet, ed. (New York: Academic Press) p. 118.

Unsöld, A. 1955, Physik der Sternatmospharën, 2nd ed. (Berlin: Springer-Verlag), see p. 433.

Valenti, J. \& Johns-Krull, C. 2001, in Magnetic Fields Across the Hertzsprung-Russell Diagram, eds. G. Mathys, S.K. Solanki, \& D.T. Wichramasinghe, ASP Conf. Ser. 248 (San Francisco: ASP), p. 179.

Van't Veer-Menneret, C. 1963, Ann. d'Ap. 26, 289.

Van't Veer-Menneret, C., Burkhart, C. \& Coupry, M. F. A\&A 203, 123.

Wahlgren, G. M., Dolk, L., Kalus, G., Johansson, S. \& Litzén, U. 2000, ApJ 539, 908.

White, S.M., Jackson, P.D. \& Kundu, M.R. 1993, AJ 105, 563.

Wolff, S. C. 1983, The A-Stars: Problems and Prospectives (Washington DC: NASA).

\section{Discussion}

Maceroni: Cluster Analysis Algorithms are usually applied in a new parameter space derived from Principal Component Analysis on the dataset (a space whose axes contain a decreasing amount of the sample variance going from the first to the last). The physical meaning of the clustering is sometimes trivial, sometimes rather difficult to extract. What is in your case the main physical parameter(s) producing the main branches of your dendrogram?

Cowley: This is a very interesting idea. We must try it! In the current work, which was never intended to be definitive, we simply considered each abundance as variable. Therefore, clusters that are close just mean the abundances are similar. We filled in missing data with "solar" values. There are other ways of dealing with data. Also, there are a variety of clustering algorithms that could be explored. Especially for the HgMn stars, it would be interesting to try to identify the different clusters or classes with stages in the development of anomalies.

KuRTz: Have you tried applying your cluster analysis to say Georges' theoretical abundances to see if the clusters look the same as the real stars, are there any examples you (can describe?) How (about) doing the theoretical data?

COWLEY: What I could say immediately from looking at the theoretical calculations is that you can see for example that when you look at some of those abundance patterns done at a fixed time with a given set of mixing parameters you get the same family that you would get if you consider one mixing parameter for different groups of ages. In other words, you can simulate the effect of a star aging by just having a different mixing 
parameter. So in that sense the cluster analysis would show you that the two methods are giving you the same or similar results. In many cases, when you look at these things, most of what you see are things that you already know. And that should you give some confidence that you are not doing something wrong. But you see a few new things. Now it could be that some other person would say "that's not anything new, I knew that all along." But some results may still be new to me, and therefore valuable for me to recognize. What I just described is an example of a case when we would see something we already knew.

VAUCLAIR: I was surprised to see so many gaps in the abundance determinations in well known stars like HR 7143, $\xi$ Lup or 32 Aqr.

COWLEY: Yes, the gaps are unfortunate. Usually it means that there are no usable lines in the regions studied. I think that abundance workers should make a spcial effort to try to fill some of these gaps.

Mathys: In the dendogram with various types of CP stars, where Przybylskyi's star stands out, is HD 965 included?

Cowley: No. But some stars are certainly more closely related to HD 101065 than in those shown.

Mathys: Could you comment about the possible presence of unstable isotopes in the atmospheres of CP stars?

COWLEY: I ran out of time. We have found evidence for some very surprising elements. Dr. Bidelman has a poster that you may peruse. For Pm II, the sepctroscopic evidence is quite strong. However, if you wish to say it is impossible, a reasonable possibility is that the atomic line lists, we used several, are contaminated with lines from other elements. The laboratory spectroscopists tell me that is unlikely. Still, it seems the easiest way for me wiggle out of an otherwise very interesting result.

AlECIAN: This is rather a comment. The building of abundance anomalies by diffusion is a non-liear process. We are not sure than a time-dependent diffusion will lead to a sationary solution (this is at least true for HgMn stars). Several stars with the same properties could then exhibit various abundance patterns. Therefore, methods of classification only based on abundance patterns are perhaps inadequate.

COWLEY: This is a comment rather than a question. 